\title{
Maximal Heart Rate in Soccer Players: Measured versus Age-predicted
}

\author{
Pantelis T. Nikolaidis
}

Background: Although maximal heart rate $\left(\mathrm{HR}_{\max }\right)$ is widely used to assess exercise intensity in sport training, and particularly in soccer, there are limited data with regards to the use of age-based prediction equations of $\mathrm{HR}_{\max }$ in soccer players. The aim of this study was to compare the measured-HR $\mathrm{max}_{\text {max }}$ with two prediction equations (Fox-HR ${ }_{\max }=220-$ age and Tanaka-HR ${ }_{\max }=208-0.7 \times$ age) in soccer players.

Methods: $\quad$ Adolescent $(n=162,15.8 \pm 1.5$ years $)$ and adult players ( $n=158,23.4 \pm 4.6$ years), all members of competitive clubs, voluntarily performed a graded exercise field test (Conconi protocol) to assess $\mathrm{HR}_{\max }$.

Results: $\quad$ The measured-HR ${ }_{\text {max }}(197.6 \pm 9.4$ bpm in total, $200.2 \pm 7.9$ bpm in adolescent players, and $195.0 \pm 10.0 \mathrm{bpm}$ in adult players) was explained by the formula $\mathrm{HR}_{\text {max }}=212.3-0.75 \times$ age $(r=0.41$, standard error of the estimate $=8.6$ ). In the total sample, Fox-HR ${ }_{\max }$ overestimated measured-HR ${ }_{\max }$ [mean difference (95\% confidence intervals $)=2.8 \mathrm{bpm}(1.6 ; 3.9)]$, while Tanaka-HR ${ }_{\max }$

\section{At a Glance Commentary}

Scientific background of the subject

Maximal heart rate is used widely to define exercise intensity in soccer. Occasionally, its measurement is not possible and then, the use of an age-based prediction equation is an alternative.

\section{What this study adds to the field}

We observed that the traditional "220-age" overestimates actual value increasing the risk for undesirable fatigue, whereas the "208-0.7 $\times$ age" underestimates actual value leading to prescribe exercise of inadequate intensity. Therefore, we highlight the need for the development of sport-specific equations. underestimated $\mathrm{HR}_{\max }[-3.3 \mathrm{bpm}(-4.5 ;-2.2)]$. In adolescents, Fox-HR ${ }_{\max }$ overestimated measured-HR ${ }_{\max }[4.0 \mathrm{bpm}(2.5 ; 5.5)]$ and Tanaka-HR ${ }_{\max }$ underestimated $\mathrm{HR}_{\max }[-3.2 \mathrm{bpm}(-4.7 ;-1.8)]$. In adults, Tanaka-HR ${ }_{\max }$ underestimated $\mathrm{HR}_{\max }[-5.0 \mathrm{bpm}(-5.3 ;-4.7)]$, while there was not any difference between Fox-HR $_{\text {max }}$ and measured-HR ${ }_{\max }[1.6 \mathrm{bpm}(-3.4 ; 0.2)]$.

Conclusions: The results of this study failed to validate two widely used prediction equations in a large sample of soccer players, indicating the need for a sport-specific equation. On the other hand, the new equation that we presented should be investigated further by future studies before being adopted by coaches and fitness trainers.

(Biomed J 2015;38:84-89)

Key words: age groups, athletes, cardiac rate, football, graded exercise test, prediction equations

Soccer training is based on the optimal prescription of exercise mode, duration, and intensity. A daily task of coaches and fitness trainers is to plan an exercise program of adequate intensity. On the other hand, special care is given to see that the exercise intensity does not increase the likelihood of overtraining. When working with soccer players, coaches and fitness trainers often establish training heart rate (HR) intensities for aerobic exercise based on maximal HR ( $\left.\mathrm{HR}_{\max }\right)$, for example, Karvonen method. ${ }^{[1]}$ $\mathrm{HR}_{\max }$ is measured as the maximal value recorded at the end of graded exercise test (GXT) either in a laboratory or in the field. However, occasionally it is not desirable to perform a GXT (e.g. to avoid the fatigue induced by maximal testing during the competitive period).

When it is not possible to measure $\mathrm{HR}_{\max }$, its prediction from an age-based equation is an alternative, which is widely

From the Department of Physical and Cultural Education, Hellenic Army Academy, Attiki, Greece Received: Dec. 30, 2013; Accepted: Apr. 14, 2014

Correspondence to: Dr. Pantelis T. Nikolaidis, Department of Physical and Cultural Education, Hellenic Army Academy, Greece. 16673 Vari, Attiki, Greece. Tel: 30-2104904023; Fax: 30-2104907557; E-mail: pademil@ hotmail.com

DOI: $10.4103 / 2319-4170.131397$ 
used by coaches and fitness trainers. Two popular equations used in the daily sport practice are those of Fox, Naughton, and Haskell (Fox-HR ${ }_{\max }=220-$ age $)^{[2]}$ and Tanaka, Monahan, and Seals (Tanaka-HR ${ }_{\max }=208-0.7 \times$ age) ${ }^{[3]}$ The validity of these equations has been examined extensively in large samples of adults ${ }^{[3-11]}$ and in specific categories of population, for example, healthy, ${ }^{[3,6]}$ sedentary, ${ }^{[4,7]}$ overweight, ${ }^{[5]}$ sportspersons, ${ }^{[8,12]}$ and individuals with mental retardation. ${ }^{[10]}$ The aforementioned studies have used a GXT in the laboratory setting to elicit $\mathrm{HR}_{\max }$. In contrast, only a few studies have been conducted in children and adolescents ${ }^{[13-15]}$ and have used a field protocol. ${ }^{[16]}$ Few studies had a longitudinal design. ${ }^{[17,18]}$

While the abovementioned studies have covered many sides of the estimation of $\mathrm{HR}_{\max }$, there are some aspects that need further research, for example, athletes and adolescents are under-represented in this body of research. In a recent study, it was shown that athletes of speed/power sports had similar measured- $\mathrm{HR}_{\max }$ with endurance athletes and both had lower values than those who were untrained. ${ }^{[19]}$ This difference between trained and untrained individuals highlights the need to examine the popular prediction equations in sport samples. In addition, the various protocols of GXT in laboratory and in field may elicit different values of $\mathrm{HR}_{\max }$. For instance, a study on soccer players revealed higher $\mathrm{HR}_{\max }$ in a field test (multistage shuttle run) than in a GXT on treadmill. ${ }^{[20]}$

Therefore, the aim of this study was to examine the validity of Fox-HR ${ }_{\max }$ and Tanaka-HR ${ }_{\max }$ in a large sample of soccer players. If differences of age-predicted-HR ${ }_{\max }$ from the measured-HR ${ }_{\max }$ were found, we presented alternative soccer-specific equations based on the actual values of players. In addition, we investigated whether these relationships vary according to the age group (adolescent vs. adult soccer players).

\section{METHODS}

\section{Study design and participants}

Three hundred twenty players from the soccer clubs in Athens were recruited to participate in this study, which was conducted for 2 days. On the first day, the participants visited the laboratory where the anthropometric measurements were taken. On the second day, within a week from the first session, they performed a GXT (Conconi protocol) in a soccer field.

\section{Protocols and equipment}

\section{Anthropometry}

Height, weight, and skinfolds were measured with subjects in minimal clothing and barefoot. An electronic weighing scale (HD-351; Tanita, Arlington Heights, IL, USA) was employed for measurement of weight (to the nearest $0.1 \mathrm{~kg}$ ), a portable stadiometer (SECA Leicester, UK) for height $(0.001 \mathrm{~m})$, and a caliper (Harpenden, West Sussex, UK) for skinfolds $(0.5 \mathrm{~mm})$. Body mass index was calculated as the quotient of body mass $(\mathrm{kg})$ to height squared $\left(\mathrm{m}^{2}\right)$, and body fat $(\mathrm{BF})$ was estimated from the sum of 10 skinfolds (cheek, wattle, chest I, triceps, subscapular, abdominal, chest II, suprailiac, thigh, and calf; $\mathrm{BF}=-41.32+12.59 \times \log _{\mathrm{e}} x$, where $x$ the sum of 10 skinfolds). ${ }^{[21]}$

\section{Graded exercise test}

A modified version of Conconi test was used to assess the maximal aerobic running speed. ${ }^{[22]}$ Briefly, after a 20-min warm-up including jogging and stretching exercises, participants performed an incremental running test in the field around a $200 \mathrm{~m}$ square. The initial speed was set at $9 \mathrm{~km} / \mathrm{h}$ and was increased every $200 \mathrm{~m}$ by $0.3-0.7 \mathrm{~km} / \mathrm{h}$ till exhaustion. During the late stages of the test, participants were cheered vigorously so that they made maximal effort. In addition, they had been instructed to adhere strictly to the speed which was determined by audio signals. Measured $\mathrm{HR}_{\max }$ was defined as the highest value attained during the test. HR was recorded continuously during the test by Team2 Pro (Polar Electro Oy, Kempele, Finland).

\section{Statistical analyses}

Statistical analyses were performed using IBM SPSS v. 20.0 (SPSS, Chicago, IL, USA). Data were expressed as mean and standard deviation (SD) of the mean. Independent Student's $t$-test was used to examine the differences between adolescent and adult players. Effect sizes (ES) for statistical differences in the $t$-test were determined using the following criteria for Cohen's d: $\mathrm{ES} \leq 0.2$, trivial; $0.2<\mathrm{ES} \leq 0.6$, small; $0.6<\mathrm{ES} \leq 1.2$, moderate; $1.2<\mathrm{ES} \leq 2.0$, large; and $\mathrm{ES}>2.0$, very large. ${ }^{[23]}$ One-way repeated measures analysis of variance (ANOVA) and a subsequent Bonferroni post-hoc test (if the differences between groups were revealed) were used to examine the differences between measured and predicted $\mathrm{HR}_{\max }$. To interpret ES for statistical differences in the ANOVA, we used eta square classified as small $\left(0.010<\eta^{2} \leq 0.059\right)$, medium $\left(0.059<\eta^{2} \leq 0.138\right)$, and large $\left(\eta^{2}>0.138\right) \cdot{ }^{[24]}$ Bland-Altman ${ }^{[25]}$ analysis was used to examine the accuracy and variability of prediction equations. Associations between measured $\mathrm{HR}_{\max }$ and age were determined using Pearson's product moment correlation coefficient $(r)$. Magnitude of correlation coefficients was considered as trivial if $r \leq 0.1$, small if $0.1 \leq r<0.3$, moderate if $0.3 \leq r<0.5$, large if $0.5 \leq r<0.7$, very large if $0.7 \leq r<0.9$, nearly perfect if $r \geq 0.9$, and perfect if $r=1 .{ }^{[26]}$ In addition, we used linear regression to model the predic- 
tion of $\mathrm{HR}_{\max }$ from age. The level of significance was set at $\alpha=0.05$.

\section{RESULTS}

The basic characteristics of the participants are presented in Table 1. Briefly, our sample comprised a similar number of adolescent (49.4\%) and adult soccer players $(50.6 \%)$. There were significant differences between age groups in age, weight, height, and BMI. Adult players were older [mean difference $(95 \%$ confidence intervals $)=7.6$ years $(6.8 ; 8.3)$, Cohen's $d=2.2$, very large ES], heavier [9.4 $\mathrm{kg}(7.4 ; 11.3), \mathrm{d}=1.0$, moderate ES], taller [ $4.5 \mathrm{~cm}(2.8 ; 6.1), \mathrm{d}=0.6$, small ES], and with higher BMI $\left[2.0 \mathrm{~kg} / \mathrm{m}^{2}(1.5 ; 2.4), \mathrm{d}=1.0\right.$, moderate ES] and maximal aerobic speed (MAS) $[0.6 \mathrm{~km} / \mathrm{h}(0.2 ; 1.0), \mathrm{d}=0.3$, small ES] than adolescent players, while there was no difference in BF $[-0.6 \%(-1.4 ; 0.2), \mathrm{d}=0.2$, small ES].

The measured-HR ${ }_{\max }$ and predicted-HR ${ }_{\text {max }}$ are given in Table 2. There was significant age difference in the mean values for measured-HR ${ }_{\max }$. Adolescents achieved higher measured-HR ${ }_{\max }$ in the endurance test [5.1 bpm $(3.1 ; 7.1), \mathrm{d}=0.6$, small ES] than the adult players. One-way repeated measures ANOVA revealed significant differences between measured-HR ${ }_{\max }, F_{0 x}-\mathrm{HR}_{\max }$, and Tanaka-HR ${ }_{\max }$ in the total sample $\left(p<0.001, \eta^{2}=0.27\right.$, large ES) and in each age group $\left(p<0.001, \eta^{2}=0.40\right.$ in adolescents and $\eta^{2}=0.18$ in adults, large ES). In the total sample, the Fox-HR ${ }_{\max }$ overestimated measured-HR $_{\text {max }}$ [2.8 bpm $\left.(1.6 ; 3.9)\right]$, while Tanaka-HR $_{\text {max }}$ underestimated $\mathrm{HR}_{\text {max }}[-3.3 \mathrm{bpm}(4.5 ;-2.2)]$. In adolescents, Fox-HR max $_{\text {ax }}$ overestimated measured-HR ${ }_{\max }[4.0$ bpm $(2.5 ; 5.5)]$ and Tanaka-HR ${ }_{\text {max }}$ underestimated $\mathrm{HR}_{\text {max }}[-3.2$ bpm $(-4.7 ;-1.8)]$. In adults, Tanaka-HR ${ }_{\text {max }}$ underestimated $\mathrm{HR}_{\text {max }}$ $[-5.0 \mathrm{bpm}(-5.3 ; 4.7)]$, while there was not any difference between Fox-HR $_{\text {max }}$ and measured-HR ${ }_{\text {max }}[1.6 \mathrm{bpm}(-3.4 ; 0.2)]$.

The relationship between measured-HR max $_{\text {and }}$ and is depicted in Figure 1. $\mathrm{HR}_{\text {max }}$ was moderately correlated with age in the total sample $(r=-0.41, p<0.001)$. The correla-

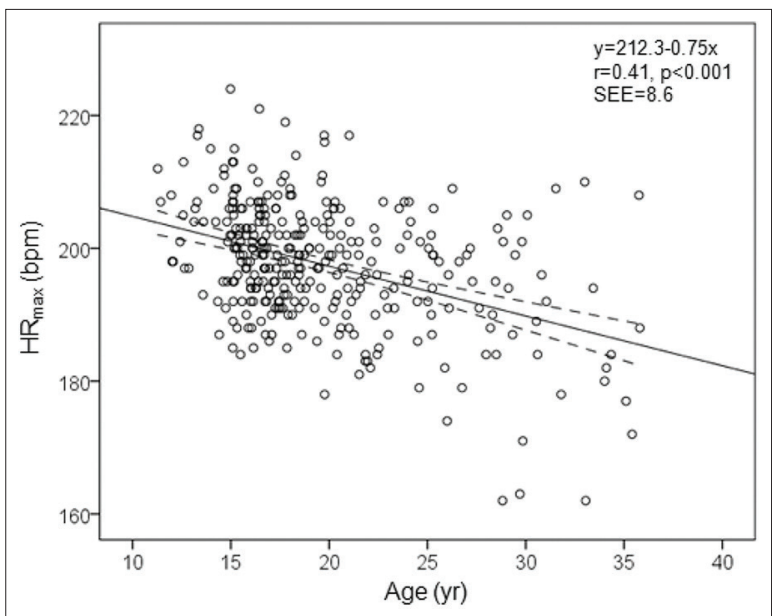

Figure 1: Relationship between age and maximal heart rate $\left(\mathrm{HR}_{\max }\right)$ in soccer players.

Table 1: Descriptive characteristics, shown as mean (standard deviation) with minimum and maximum values of the participants by age group

\begin{tabular}{lcccc}
\hline & Total $(N=320)$ & Adolescents $(n=158)$ & Adults $(n=162)$ & Comparison \\
\hline Anthropometry & & & & \\
Age (years) & $19.7(5.1), 11.3-35.8$ & $15.8(1.5), 11.3-18.0$ & $23.4(4.6), 18.0-35.8$ & $\mathrm{t}_{318}=-19.6, P<0.001$ \\
Weight $(\mathrm{kg})$ & $69.9(10.1), 36.0-98.7$ & $65.1(10.0), 36.0-88.2$ & $74.5(8.0), 57.0-98.7$ & $\mathrm{t}_{318}=-9.3, P<0.001$ \\
Height $(\mathrm{cm})$ & $176.2(7.7), 139.7-194.0$ & $173.9(8.4), 139.7-190.0$ & $178.3(6.1), 164.6-194.0$ & $\mathrm{t}_{318}=-5.4, P<0.001$ \\
BMI $\left(\mathrm{kg} / \mathrm{m}^{2}\right)$ & $22.4(2.2), 16.7-29.8$ & $21.4(2.1), 16.7-27.7$ & $23.4(1.9), 18.2-29.8$ & $\mathrm{t}_{318}=-8.7, P<0.001$ \\
BF $(\%)$ & $14.8(3.7), 5.9-26.5$ & $15.1(3.9), 7.8-26.5$ & $14.5(3.5), 5.9-25.0$ & $\mathrm{t}_{318}=1.5, P=0.133$ \\
Conconi test & & & & \\
Duration $(\mathrm{min}: \mathrm{s})$ & $13: 17(2: 21), 3: 45-19: 24$ & $12: 52(2: 42), 3: 45-19: 24$ & $13: 41(1: 54), 4: 30-17: 30$ & $\mathrm{t}_{318}=-3.1, P=0.002$ \\
MAS $(\mathrm{km} / \mathrm{h})$ & $15.6(1.8), 10.9-20.0$ & $15.3(2.0), 10.9-20.0$ & $15.9(1.6), 10.9-19.5$ & $\mathrm{t}_{318}=-2.9, P=0.003$ \\
\hline Abbris & & &
\end{tabular}

Abbreviations: BMI: Body mass index; BF: Body fat; MAS: Maximal aerobic speed

Table 2: Measured-HR ${ }_{\max }$ and age-predicted $\mathrm{HR}_{\max }$, shown as mean (standard deviation) with minimum and maximum values of the participants by age group

\begin{tabular}{|c|c|c|c|}
\hline & Total $(N=320)$ & Adolescents $(n=158)$ & Adults $(n=162)$ \\
\hline Measured-HR ${ }_{\max }(\mathrm{bpm})$ & $197.6(9.4), 162$ to 224 & $200.2(7.9), 184-224$ & $195.0(10.0), 162$ to 217 \\
\hline Fox-HR $_{\max }(\mathrm{bpm})$ & $200.3(5.1)^{*}, 184.2$ to 208.7 & $204.2(1.5)^{*}, 202.0$ to 208.7 & $196.6(4.6), 184.2$ to 202.0 \\
\hline Tanaka-HR $_{\max }(\mathrm{bpm})$ & $194.2(3.6)^{*}, 182.9$ to 200.1 & $196.9(1.0)^{*}, 195.4$ to 200.1 & $191.6(3.2)^{*}, 182.9$ to 195.4 \\
\hline Fox-HR ${ }_{\max }-$ measured-HR $\max _{\max }(\mathrm{bpm})$ & $2.8(8.7),-23.8$ to 29.2 & $4.0(7.7),-19.0$ to 20.5 & $1.6(9.4),-23.8$ to 29.2 \\
\hline Tanaka-HR $_{\max }$ measured-HR ${ }_{\max }(\mathrm{bpm})$ & $-3.3(8.6),-26.5$ to 25.8 & $-3.2(7.7),-26.5$ to 13.2 & $-3.4(9.4),-25.1$ to 25.8 \\
\hline
\end{tabular}

*Statistically differed from measured $\mathrm{HR}_{\max }(p<0.001)$ 
tions for adolescent and adult players were low $(r=-0.27$, $p=0.001)$ and moderate $(r=-0.36, p<0.001)$, respectively. The measured-HR ${ }_{\text {max }}$ was explained by the formula $\mathrm{HR}_{\text {max }}=212.3-0.75 \times$ age (standard error of the estimate, $\mathrm{SEE}=8.6)$ in the total sample, $\mathrm{HR}_{\max }=223-1.44$ age $(\mathrm{SEE}=7.6)$ in adolescent players, and $\mathrm{HR}_{\max }=213.2-0.78 \times$ age $(\mathrm{SEE}=9.4)$ in adult players.

Figures 2 and 3 show the Bland-Altman plots of the difference between predicted- $\mathrm{HR}_{\max }$ and measured-HR $\mathrm{H}_{\max }$ in total and in each age group for Fox-HR ${ }_{\max }$ and Tanaka-HR ${ }_{\max }$, respectively. In the case of Fox equation, we observed that there was overestimation of $\mathrm{HR}_{\max }$ at low values of $\mathrm{HR}_{\max }$ and underestimation of $\mathrm{HR}_{\max }$ at high values of $\mathrm{HR}_{\max }$. A similar trend was also noticed in the case of Tanaka equation.

\section{DISCUSSION}

The main finding of this study was that neither Fox nor Tanaka equation provides accurate values of $\mathrm{HR}_{\max }$ in the total sample of soccer players. In the total sample and in adolescent players, Fox-HR ${ }_{\text {max }}$ overestimated measured-HR ${ }_{\text {max }}$, while Tanaka-HR max $_{\text {ax }}$ underestimated $\mathrm{HR}_{\text {max }}$ in each age group. We did not find any difference between measured-HR ${ }_{\max }$ and Fox-HR $\mathrm{Hax}_{\max }$ in adult players.

Our study did not confirm the findings of previous research supporting that Fox-HR max $_{\text {max }}$ underestimates $\mathrm{HR}_{\text {max }}$ with increasing age ${ }^{[3,17]}$ which could be attributed to the younger age of the participants in the present study. In contrast, our findings confirmed that Fox- $\mathrm{HR}_{\max }$ overestimates $\mathrm{HR}_{\max }$ in adolescents. ${ }^{[15]}$

The basic characteristics of the participants were similar to those reported recently, ${ }^{[27]}$ and the differences in weight, height, BMI, and endurance between adolescent and adult players were in line with previous research. ${ }^{[27,28]}$ Comparison between age groups with regard to their mean $\mathrm{HR}_{\max }$ revealed lower values in the older group ( $\sim 5 \mathrm{bpm})$, a finding which is in accordance with the negative correlation between $\mathrm{HR}_{\max }$ and age. Compared with adult players (17.8 years), this relationship had a lower magnitude in adolescent players, which is attributed to the shorter span of chronological age in this group (6.7 years).

Similarly, the variation in the span of chronological age may explain the discrepancy between this study and previous research with regard to the above-mentioned correlation. In a previous study covering a relatively short span of ages (10-16 years), the correlation between $\mathrm{HR}_{\max }$ and age was $-0.10,{ }^{[15]}$ while in studies with large span, we observed large to very large correlations (e.g. $15-75$ years, $r=-0.56$; ${ }^{[8]}$ $14-77$ years, $r=-0.60 ;{ }^{[9]} 16-71$ years, $r=-0.67 ;{ }^{[29]} 19-89$ years, $r=-0.60 ; ;^{[6]} 16-65$ years, $r=-0.60 ; ;^{[4]} 18-81$ years, $\left.r=-0.79^{[3]}\right)$.

Compared with boys of similar age (10-16 years $)^{[15]}$ who performed a GXT on treadmill, the adolescent soccer

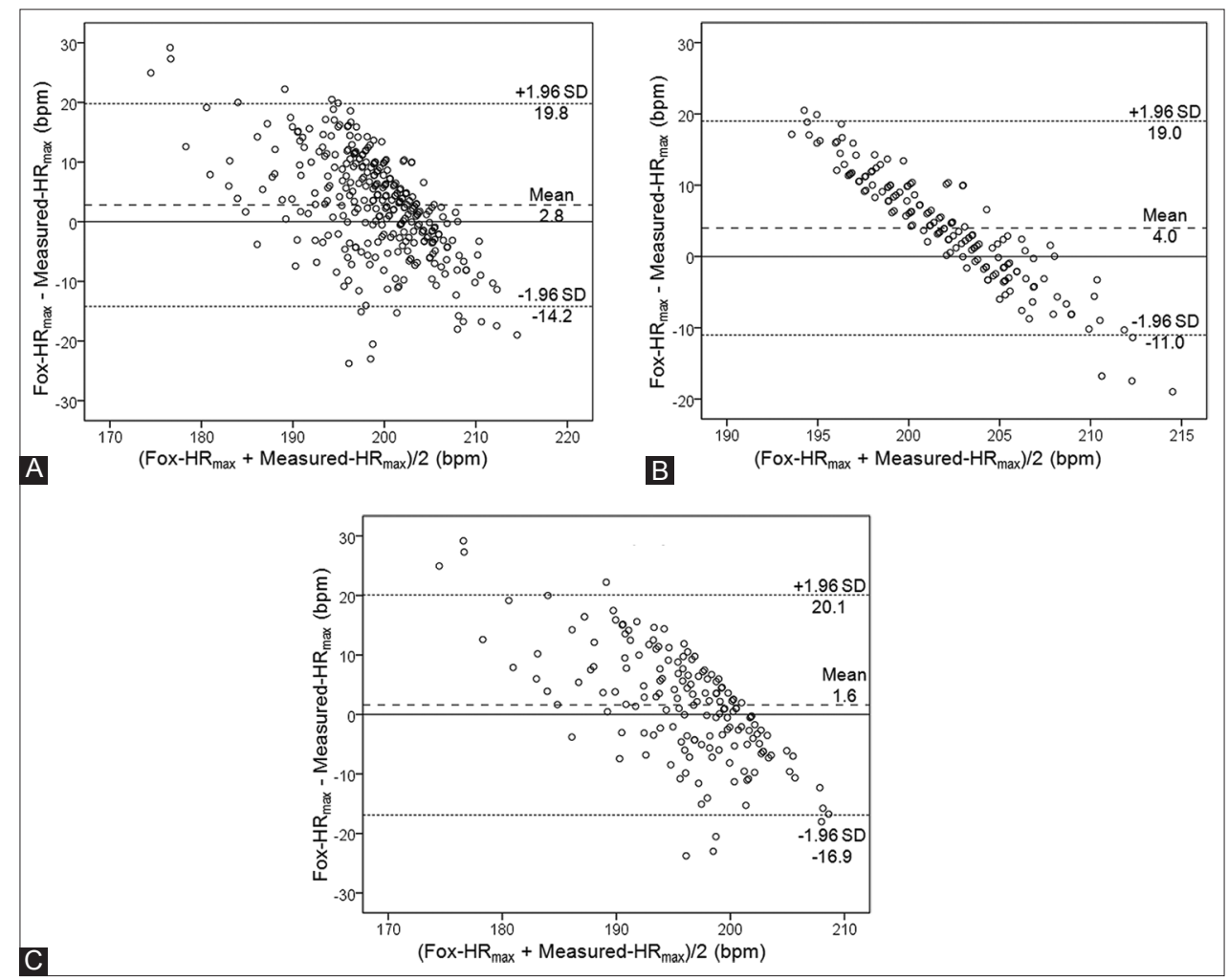

Figure 2: Bland-Altman plots of the difference between Fox-HR ${ }_{\max }$ and measured-HR max $_{\text {in }}$ the total sample (A), in adolescent players (B), and in adult soccer players $(\mathrm{C})$. 


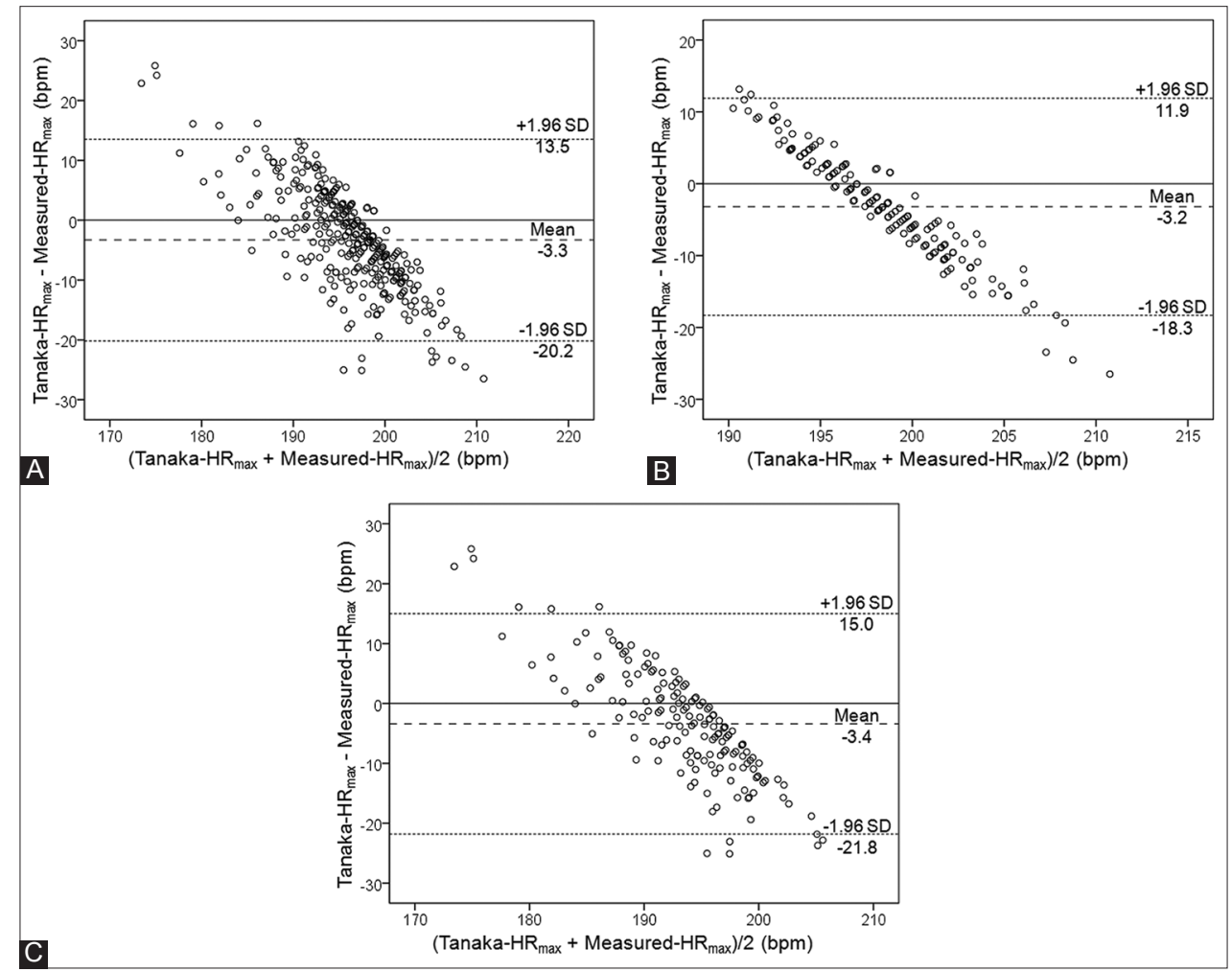

Figure 3: Bland-Altman plots of the difference between Tanaka-HR ${ }_{\max }$ and measured-HR ${ }_{\max }$ in the total sample (A), in adolescent players (B), and in adult soccer players $(\mathrm{C})$.

Table 3: Summary of the main findings

\begin{tabular}{|c|c|c|}
\hline Total sample & Adolescent players & Adult players \\
\hline $\begin{array}{l}\text { Fox-HR } \mathrm{Hx}_{\mathrm{m}}>\text { measured-HR } \mathrm{max}_{\max }>\text { Tanaka-HR } \\
\text { New equations based on the present study }\end{array}$ & Fox-HR $_{\text {mx }}>$ measured-HR max $_{\text {max }}>$ Tanaka-HR ${ }_{\max }$ & Fox-HR ${ }_{\mathrm{mx}}=$ measured $-\mathrm{HR}_{\max }>$ Tanaka-HR $\mathrm{Hax}_{\max }$ \\
\hline $\mathrm{HR}_{\max }=212.3-0.75 \times$ age $(r=-0.41, \mathrm{SEE}=8.6)$ & $\mathrm{HR}_{\max }=223-1.44 \times$ age $(r=-0.27, \mathrm{SEE}=7.6)$ & $\mathrm{HR}_{\max }=213.2-0.78 \times$ age $(r=-0.36, \mathrm{SEE}=9.4)$ \\
\hline
\end{tabular}

players in the present study achieved higher $\mathrm{HR}_{\text {max }}(\sim 4 \mathrm{bpm})$. In contrast, we found similar values with another study on individuals aged 7-17 years. ${ }^{[13]}$ In addition, the adult players of our sample achieved higher $\mathrm{HR}_{\text {max }}(\sim 11.5 \mathrm{bpm}$ and $\sim 10 \mathrm{bpm}$ ) than adults of similar age who performed a GXT on cycle ergometer in two previous studies. ${ }^{[30,31]} \mathrm{A}$ possible explanation of these differences might be the use of different GXT protocols and the biological variation.

The main limitation of this study is that it presents the common drawbacks of any field GXT; in contrast to the criteria of maximal effort (e.g. plateau of oxygen uptake, $\mathrm{HR}>90-95 \%$ of $\mathrm{HR}_{\max }$, respiratory quotient $>1.15$, and lactate $>9-10 \mathrm{mmol} / \mathrm{L}^{[32]}$ ) used typically in a laboratory setting, the only criterion used to evaluate participants' effort was their oral confirmation that they had run till exhaustion. In addition, we examined only the relationship between $\mathrm{HR}_{\max }$ and age, and not the effects of other confounders on this relationship. It has been suggested that the overestimation of $\mathrm{HR}_{\max }$ might be associated with increased weight and smoking, while its underestimation might be associated with rest HR. ${ }^{[9]}$ Despite these drawbacks, our findings have important practical implications for soccer training as well as for clinical practice, where HR is measured frequently. ${ }^{[33,34]}$ To the best of our knowledge, this is the first study to examine the validity of two popular prediction equations of $\mathrm{HR}_{\max }$ in a large sample of soccer players including both adolescents and adults. Moreover, we have presented new soccer-specific equations that might be of use for coaches and trainers in the future [Table 3].

\section{Conclusions}

The results of this study failed to validate two widely used prediction equations of $\mathrm{HR}_{\max }$ in a large sample of soccer players, indicating the need for a sport-specific equation. Although Tanaka equation offered better estimates of $\mathrm{HR}_{\text {max }}$ in non-athletes than the older equation of Fox, its use in soccer players systematically underestimated $\mathrm{HR}_{\max }$; therefore, its application in this sport might lead to prescribe exercise 
of inadequate intensity. On the other hand, the new equation that we presented should be investigated further by future studies before being adopted by coaches and fitness trainers. An important issue when examining the validity of various equations or comparing $\mathrm{HR}_{\max }$ values is to take into account the GXT protocol being used.

\section{REFERENCES}

1. Karvonen MJ, Kentala E, Mustala O. The effects of training on heart rate; a longitudinal study. Ann Med Exp Biol Fenn 1957;35:307-15.

2. Fox SM $3^{\text {rd }}$, Naughton JP, Haskell WL. Physical activity and the prevention of coronary heart disease. Ann Clin Res 1971;3:404-32.

3. Tanaka H, Monahan KD, Seals DR. Age-predicted maximal heart rate revisited. J Am Coll Cardiol 2001;37:153-6.

4. Sarzynski MA, Rankinen T, Earnest CP, Leon AS, Rao DC, Skinner JS, et al. Measured maximal heart rates compared to commonly used age-based prediction equations in the heritage family study. Am J Hum Biol 2013;25:695-701.

5. Franckowiak SC, Dobrosielski DA, Reilley SM, Walston JD, Andersen RE. Maximal heart rate prediction in adults that are overweight or obese. J Strength Cond Res 2011;25:1407-12.

6. Nes BM, Janszky I, Wisløff U, Støylen A, Karlsen T. Age-predicted maximal heart rate in healthy subjects: The HUNT Fitness Study. Scand J Med Sci Sports 2013;23:697-704.

7. Camarda SR, Tebexreni AS, Páfaro CN, Sasai FB, Tambeiro VL, Juliano Y, et al. Comparison of maximal heart rate using the prediction equations proposed by Karvonen and Tanaka. Arq Bras Cardiol 2008;91:311-4.

8. Lester M, Sheffield LT, Trammell P, Reeves TJ. The effect of age and athletic training on the maximal heart rate during muscular exercise. Am Heart J 1968;76:370-6.

9. Whaley MH, Kaminsky LA, Dwyer GB, Getchell LH, Norton JA. Predictors of over- and underachievement of age-predicted maximal heart rate. Med Sci Sports Exerc 1992;24:1173-9.

10. Fernhall B, McCubbin JA, Pitetti KH, Rintala P, Rimmer JH, Millar AL, et al. Prediction of maximal heart rate in individuals with mental retardation. Med Sci Sports Exerc 2001;33:1655-60.

11. Balassiano DH, de Araújo CG. Maximal heart rate: Influence of sport practice during childhood and adolescence. Arq Bras Cardiol 2013;100:333-8.

12. Faff J, Sitkowski D, Ladyga M, Klusiewicz A, Borkowski L, Starczewska-Czapowska J. Maximal heart rate in athletes. Biol Sport 2007;24:129-42.

13. Mahon AD, Marjerrison AD, Lee JD, Woodruff ME, Hanna LE. Evaluating the prediction of maximal heart rate in children and adolescents. Res Q Exerc Sport 2010;81:466-71.

14. Colantonio E, Kiss MA. Is the HRmax $=220$-age equation valid to prescribe exercise training in children? J Exerc Physiol Online 2013;16:19-27.

15. Machado FA, Denadai BS. Validity of maximum heart rate prediction equations for children and adolescents. Arq Bras Cardiol 2011;97:136-40.

16. Cleary MA, Hetzler RK, Wages JJ, Lentz MA, Stickley CD, Kimura IF. Comparisons of age-predicted maximum heart rate equations in college-aged subjects. J Strength Cond Res 2011;25:2591-7.

17. Gellish RL, Goslin BR, Olson RE, McDonald A, Russi GD, Moudgil VK. Longitudinal modeling of the relationship between age and maximal heart rate. Med Sci Sports Exerc 2007;39:822-9.

18. Zhu N, Suarez-Lopez JR, Sidney S, Sternfeld B, Schreiner PJ, Carnethon MR, et al. Longitudinal examination of age-predicted symptom-limited exercise maximum HR. Med Sci Sports Exerc 2010;42:1519-27.

19. Kusy K, Zielinski J. Aerobic capacity in speed-power athletes aged $20-90$ years vs endurance runners and untrained participants. Scand J Med Sci Sports 2014;24:68-79.

20. Aziz AR, Tan FH, Teh KC. A pilot study comparing two field tests with the treadmill run test in soccer players. J Sports Sci Med 2005;4:105-12.

21. Parizkova J. Lean body mass and depot fat during autogenesis in humans. In: Parizkova J, Rogozkin V, editors. Nutrition, Physical Fitness and Health: International Series on Sport Sciences. Baltimore: University Park Press; 1978.

22. Conconi F, Ferrari M, Ziglio PG, Droghetti P, Codeca L. Determination of the anaerobic threshold by a noninvasive field test in runners. J Appl Physiol Respir Environ Exerc Physiol 1982;52:869-73.

23. Batterham AM, Hopkins WG. Making meaningful inferences about magnitudes. Int J Sports Physiol Perform 2006;1:50-7.

24. Cohen J. Statistical power analysis for the behavioral sciences, $2^{\text {nd }} e d$. Hillsdale, NJ: Lawrence Erlbaum Associates; 1988.

25. Bland JM, Altman DG. Statistical methods for assessing agreement between two methods of clinical measurement. Lancet 1986;1:307-10.

26. Hopkins WG. A Scale of Magnitudes for Effect Statistics. Available from: http://wwwsportsciorg/resource/stats/indexhtml. 2002. [Last accessed on 2009 Mar 20].

27. Nikolaidis PT, Vassilios Karydis N. Physique and body composition in soccer players across adolescence. Asian J Sports Med 2011;2:75-82.

28. Nikolaidis PT. Cardiorespiratory power across adolescence in male soccer players. Fiziol Cheloveka 2011;37:137-42.

29. Inbar O, Oren A, Scheinowitz M, Rotstein A, Dlin R, Casaburi R. Normal cardiopulmonary responses during incremental exercise in 20- to 70-yr-old men. Med Sci Sports Exerc 1994;26:538-46.

30. Bouzas Marins JC, Delgado Fernández M, Benito Peinado PJ. Acuracy of different equation to predict maximal heart rate in cycle ergometer. Arch Med Deporte 2013;30:14-20.

31. Fairbarn MS, Blackie SP, McElvaney NG, Wiggs BR, Pare PD, Pardy RL. Prediction of heart rate and oxygen uptake during incremental and maximal exercise in healthy adults. Chest 1994;105:1365-9.

32. Howley ET, Bassett DR Jr, Welch HG. Criteria for maximal oxygen uptake: Review and commentary. Med Sci Sports Exerc 1995;27:1292-301.

33. Cheng JH, Hua CC, Chen NH, Liu YC, Yu CC. Autonomic activity difference during continuous positive airway pressure titration in patients with obstructive sleep apnea/hypopnea syndrome with or without hypertension. Chang Gung Med J 2011;34:410-7.

34. Wu KP, Lai PL, Lee LF, Hsu CC. Autonomic dysreflexia triggered by an unstable lumbar spine in a quadriplegic patient. Chang Gung Med J 2005;28:508-11. 